\title{
Fibronectin 1 inhibits the apoptosis of human trophoblasts by activating the PI3K/Akt signaling pathway
}

\author{
JINLONG JI ${ }^{1,2}$, LIPING CHEN ${ }^{2}$, YANYAN ZHUANG ${ }^{1}$, YUN HAN $^{2}$, WEICHUN TANG $^{2}$ and FEI XIA ${ }^{1}$ \\ ${ }^{1}$ Department of Gynecology and Obstetrics, The First Affiliated Hospital of Soochow University, \\ Suzhou, Jiangsu 215006; ${ }^{2}$ Department of Gynecology and Obstetrics, The Second Affiliated Hospital \\ of Nantong University, Nantong, Jiangsu 226001, P.R. China
}

Received December 8, 2019; Accepted April 27, 2020

DOI: $10.3892 /$ ijmm.2020.4735

\begin{abstract}
The excessive apoptosis of human trophoblasts can cause pregnancy-related diseases. It has been reported that fibronectin 1 (FN1) is closely associated with the invasion of human trophoblasts. The aim of the present study was to examine the effects of FN1 on the apoptosis of human trophoblasts and to investigate the underlying molecular mechanisms. It was found that FN1, a differentially expressed gene (DEG) in the GSE127170 dataset, was identified as the hub gene in a protein-protein interaction (PPI) network generated using the cytoHubba plug-in of Cytoscape software. The Metascape website was used to perform GO enrichment analysis, and the Kyoto Encyclopedia of Genes and Genomes (KEGG) pathway database was used to perform KEGG pathway analysis. Experimental analyses revealed that FN1 expression was downregulated in the chorionic villus tissues of patients diagnosed with and mice subjected to spontaneous abortion (SA). CCK-8 and flow cytometric assays revealed that the knockdown of FN1 decreased the viability and promoted the apoptosis of JEG-3 and BeWo cells. In vivo experiments demonstrated that the knockdown of FN1 promoted the apoptosis of trophoblasts in the chorionic villus tissues obtained from mice subjected to SA, whereas FN1 overexpression increased cell viability and inhibited cell apoptosis. The protein levels of cleaved caspase-3 and Bax were increased by the silencing of FN1 and decreased by FN1 overexpression. The protein expression levels of Bcl-2, proliferating cell nuclear antigen (PCNA) and Ki67 were decreased by the silencing of FN1; however, the overexpression of FN1 increased these levels. The results of western blot analysis revealed that the knockdown of FN1 inhibited the PI3K/Akt signaling pathway, while the overexpression of FN1 activated the PI3K/Akt signaling pathway. Consistently, the apoptosis-inhibiting effect of FN1 overexpression was
\end{abstract}

Correspondence to: Professor Fei Xia, Department of Gynecology and Obstetrics, The First Affiliated Hospital of Soochow University, 188 Shizi Street, Suzhou, Jiangsu 215006, P.R. China E-mail: xiafeixf1819@163.com

Key words: fibronectin 1, apoptosis, trophoblasts, PI3K/Akt reversed by a PI3K/Akt signaling pathway inhibitor, and the apoptosis-promoting effect of FN1 silencing was reversed by a PI3K/Akt signaling pathway activator. On the whole, the findings of the present study demonstrate that the inhibition of FN1 induces the apoptosis of JEG-3 and BeWo cells, and the overexpression of FN1 inhibits cell apoptosis by activating the PI3K/Akt signaling pathway.

\section{Introduction}

Trophoblasts are a specific type of placental cells, that play important roles in embryo implantation and in the formation of the maternal-fetal interface (1). After the blastocyst implants into the endometrium, trophoblasts differentiate into extravillous and chorionic trophoblasts. Extravillous trophoblasts play a key role in invading the decidual stroma and blood vessels of the uterus (2). There are two types of chorionic trophoblasts: Syncytiotrophoblasts in the outer layer and cytotrophoblasts in the inner layer (3). Syncytiotrophoblasts are located on the surface of the chorion and directly participate in the material exchange between the mother and fetus (4). Cytotrophoblasts, which are located in the inner layer, have a greater proliferative ability and under certain conditions, can differentiate into extravillous trophoblasts and syncytiotrophoblasts. The dynamic balance between trophoblast proliferation and apoptosis is critical for maintaining pregnancy (5), and the disruption of this balance can lead to complications, such as preeclampsia or abortion. Studies in China and abroad have demonstrated that the trophoblast apoptotic index is significantly higher in cases of spontaneous abortion (SA) than in normal pregnancies (6-9).

Fibronectin 1 (FN1) is a high molecular weight glycoprotein that has numerous biological functions (10). FN1 is present in the extracellular matrix and plays key roles in cell adhesion, growth, migration and differentiation, and is involved in maintaining cell morphology (11). It is also a major blood protein, and functions as a crucial non-specific modulin in tissue repair, and promotes the survival of neurons in ischemic brains. FN1 binds to cells in the salivary gland and regulates their molecular structure by binding to collagen, fibrinogen and fibrin (12-14). In addition, FN1 exhibits chemotactic activity and attracts monocytes, and is involves in both blood coagulation and wound healing (11). FN1-knockout 
mice have an embryonic lethal phenotype that is characterized by severe cardiac and vascular disorders (15), indicating that FN1 is essential for embryonic development. As FN1 is highly expressed in tumor vessels and mediates angiogenesis during tumorigenesis, it also plays important roles in cancer progression. It has been reported that FN1 is highly expressed in breast cancer (16), nasopharyngeal carcinoma (17), oral squamous cell carcinoma (11) and thyroid carcinoma (18), and plays a regulatory role in the major inflammatory cells present in the tumor microenvironment. In addition, FN1 expression is significantly upregulated in uterine leiomyoma and is associated with trophoblast invasion, uterine proliferation and adhesion function (19). However, the effects of FN1 on trophoblasts and the underlying molecular mechanisms remain unknown.

\section{Materials and methods}

Data collection. The gene expression profiles of the GSE127170 dataset were obtained from the GEO database (https://www. ncbi.nlm.nih.gov/). The GSE127170 dataset includes data from 12 samples from the Agilent GPL10739 platform (Affymetrix Human Genome U133 Plus 2.0 Array). It includes replicates $(n=3)$ for the following 3 groups: Untreated JEG-3 cells vs. forkolin-treated JEG-3 cells (gene set-1), untreated BeWo cells vs. forkolin-treated BeWo cells (gene set-2), and untreated JEG-3 and BeWo cells vs. forkolin-treated JEG-3 and BeWo cells (gene set-3).

Screening results of differentially expressed genes (DEGs). The series matrix file dataset of GSE127170 was downloaded, and the gene probes of the platform were subsequently transformed to gene names by referencing the GPL10739 platform. Both the normalization of the data and the screening of the DEGs were performed using the 'limma' package in $\mathrm{R}$ language (version 3.5.1). To identify DEGs, the screening criteria were set to filter genes with a fold change (FC) value of $(\log 2 \mathrm{FCl})>1$ and a P-value of $<0.05$.

Construction of the PPI network and identification of hub genes. STRING is a public database that contains interactions between known and predicted proteins (https://string-db.org/). PPI is essential for examining protein function since it is helpful for elucidating the regulatory functions among proteins. The top 300 DEGs of the GSE127170 dataset (gene set1-3) were uploaded onto the STRING website to obtain the interrelations between proteins and to set the minimum required interaction score to 0.15 to visualize the interaction networks with Cytoscape (version 3.7.2). cytoHubba, the plug-in of Cytoscape, was used to screen the hub genes based on the 'Degree' and 'MCC' method.

GO and KEGG pathway analyses for the identification of overlapping DEGs. The overlapping DEGs from gene set1, gene set 2 and gene set 3 were obtained using the Draw Venn Diagram (http://bioinformatics.psb.ugent.be/webtools/Venn/) online tool. The Metascape website (http://metascape. org/gp/index.html\#/main/step1) was used to perform GO enrichment analysis. KEGG pathway database (https://www. kegg.jp/kegg/pathway.html) was used to perform KEGG pathway analysis. P-values of $<0.05$ were considered to indicate statistically significant differences.

Chorionic villus specimens. Chorionic villus tissue specimens from 65 patients diagnosed with SA (mean age, $26.48 \pm 3.91$ years) and 65 patients with induced abortion (IA; mean age, 27.41 \pm 2.86 years) (termed normal intrauterine early pregnancy) were obtained between January, 2015 and December, 2018 at The First Affiliated Hospital of Soochow University. The clinical information pertaining to the patients diagnosed with SA is presented in Table I. Each patient was examined by two pathologists and chorionic villus tissue was confirmed based on the histopathological assessment. Following retrieval, each tissue specimen was snap-frozen in liquid nitrogen immediately and preserved at $-80^{\circ} \mathrm{C}$ until further experimentation. The study protocol was approved by the Ethics Committee of the First Affiliated Hospital of Soochow University. A written informed consent was obtained for each sample, which was then analyzed anonymously. The present study was performed in accordance with the Declaration of Helsinki.

Inclusion and exclusion criteria. The inclusion criteria in the present study were as follows: i) A positive result for urine human chorionic gonadotropin (HCG); ii) B-ultrasound exhibited an embryonic heartbeat; iii) patients did not take hormonal drugs prior to surgery. The exclusion criteria were as follows: i) Abnormal pregnancy; ii) intrauterine device; iii) genitalia infection and uterine malformation.

Animal models of SP. For the purposes of the experiment, 8- to 10-week-old C57BL/6 male mice, C57BL/6 female mice and FN1 gene knockout $\left(\mathrm{FN}^{-/}\right) \mathrm{C} 57 \mathrm{~B} / 6 \mathrm{~L}$ female mice were purchased from Shanghai Slac Laboratory Animal Co., Ltd. The mice were housed in an environment controlled for temperature $\left(22-24^{\circ} \mathrm{C}\right)$ and conditions of light $(12 \mathrm{~h}$ light and $12 \mathrm{~h}$ darkness), with free access to standard mouse food and water. A total of 2 female mice were caged with 1 male mouse every other day, and vaginal plugs were examined the following morning as a sign of mating behavior. The day of plug detection was defined as embryonic day (E) 0.5. The pregnant mice were randomly divided into the normal pregnancy mouse group (NP), the SA mouse group (SA), the normal pregnancy $\mathrm{FN}^{-/-}$mouse group $\left(\mathrm{FN}^{-/-}\right)$and the $\mathrm{SA} \mathrm{FN1} 1^{-/-}$mouse group $\left(\mathrm{FN}^{-/}{ }_{-} \mathrm{SA}\right)$, with 6 mice in each group. Lipopolysaccharide (LPS; Sigma-Aldrich; Merck KGaA) at a dose of $2.5 \mu \mathrm{g}$ dissolved in saline was intraperitoneally injected into the pregnant C57BL/6 female mice or $\mathrm{FN}^{-1-} \mathrm{C} 57 \mathrm{BL} / 6$ female mice at $\mathrm{E} 7.5$ to induce abortion, as previously described (20). Mice in the control group received the same volume of saline as the vehicle. At E10.5, the mice were sacrificed, and the embryo resorption rate were calculated by dividing the number of resorbed embryos by the number of implantations, and the decidua were collected for use in further experiments. All the experiments involving animals in present study were carried out in accordance with the protocols of the Guidelines for the Care and Use of Laboratory Animals published by the United States National Institutes of Health (NIH Publication, revised 2011) and the Guidelines for the Care and Use of Laboratory Animals of the Chinese Animal Welfare 
Table I. Association between FN1 expression and clinical characteristics of patients diagnosed with SA.

\begin{tabular}{lcccc}
\hline & \multicolumn{4}{c}{ FN1 expression } \\
\cline { 2 - 4 } & $\begin{array}{c}\text { Cases } \\
(\mathrm{n}=65)\end{array}$ & $\begin{array}{c}\text { High } \\
(\mathrm{n}=29)\end{array}$ & $\begin{array}{c}\text { Low } \\
(\mathrm{n}=36)\end{array}$ & P-value \\
\hline Age (years) & & & & 0.314 \\
$\geq 22$ and $<30$ & 16 & 9 & 7 & \\
$\geq 30$ and $<35$ & 20 & 10 & 10 & \\
$\geq 35$ & 29 & 10 & 19 & \\
Pregnancy cycle (weeks) & & & & 0.718 \\
$\leq 6$ & 33 & 14 & 19 & \\
$>6$ and $\leq 10$ & 32 & 15 & 17 & \\
\hline
\end{tabular}

Data were analyzed using the $\chi^{2}$ test. No significant differences were observed $(\mathrm{P}>0.05)$.

Committee. The procedures were approved by the Animal Use Committees of Soochow University.

Immunohistochemistry (IHC). For human, IHC staining was performed for chorionic villus tissue specimen from IA group and SA group. For mice, IHC staining was performed for chorionic villus tissue specimen from the NP group, SA group, $\mathrm{FN}^{-/}$group and $\mathrm{FN}^{-{ }_{-}}$SSA group. Each section of chorionic villus tissue specimen from the patients was subjected to overnight incubation with one of the following primary antibodies, including anti-FN1 (1:500 dilution; ab2413, Abcam), and each section of chorionic villus tissue specimen form mice was subjected to overnight incubation with one of the following primary antibodies, including anti-cleaved caspase-3 (1:500 dilution; ab2302, Abcam), at $4^{\circ} \mathrm{C}$. Each section was then incubated in PBS and washed with PBS thrice at $37^{\circ} \mathrm{C}$. Subsequently, each section was incubated with goat anti-rabbit immunoglobulin G secondary antibody (1:1,000; cat. no. BS10043; Bioworld Technology, Inc.) at $37^{\circ} \mathrm{C}$ for 60 min. The Olympus BX51 light microscope (Olympus Corporation; magnification, $\mathrm{x} 100$ ) was used to examine the sections following IHC staining. All specimens were then assigned with scores based on the cytoplasmic staining intensity (with 0 indicating no staining; 1 indicating weak staining, 2 suggesting moderate staining, and 3 representing strong staining), and the extent of stained cells (0 indicating $0 \%, 1$ suggesting 1-24\%, 2 representing $25-49 \%, 3$ was indicative of $50-74 \%$, and 4 being indicative of $75-100 \%$ ). Moreover, the intensity score was multiplied with the score of the stained cell extent to determine the eventual immunoreactive score, which ranged from 0 (minimal) to 12 (maximal). In addition, $0,1-6$, and $\geq 8$ points were defined as negative, weak positive and strong positive expression, separately. Each experiment was carried out in triplicate.

Histopathological evaluation. The chorionic villus tissues form patients or mice were immersed in normal $10 \%$ neutral buffered formalin. The sections (5- $\mu \mathrm{m}$-thick) were cut after paraffin embedding and stained with hematoxylin and eosin (H\&E) at $37^{\circ} \mathrm{C}$ for $2 \mathrm{~min}$ according to a previous description (21). You can analysis the pathological changes under a light microscope.

Cells and cell culture. The human choriocarcinoma cell lines, JEG-3 (cat. no. HTB-36, ATCC) and BeWo (cat. no. CCL-98, ATCC) were cultured in Dulbecco's modified Eagle's medium (DMEM), $10 \%$ (v/v) fetal bovine serum (FBS), $100 \mu \mathrm{g} / \mathrm{ml}$ penicillin, $100 \mu \mathrm{g} / \mathrm{ml}$ streptomycin (Invitrogen; Thermo Fisher Scientific, Inc.) at $37^{\circ} \mathrm{C}$ in a humidified atmosphere with $5 \% \mathrm{CO}_{2}$. The cells were harvested and seeded in 6-well plates at $2 \times 10^{5}$ cells/well.

Cell transfection. The siRNA sequences targeting FN1 were synthesized from Shanghai Gene-Pharma Co. Nonsense siRNA was used as the negative control. The siRNA-FN1 sequence was as follows: 5'-TACGAATCCCCAGGCCCC GGGCCCG-3'. siRNA- FN1 (40 nm) was transfected into the JEG-3 and BeWo cells using Lipofectamine 2000 (Invitrogen; Thermo Fisher Scientific, Inc.) to reduce the mRNA and protein levels of FN1. The FN1 cDNA sequence was cloned into the pcDNA3.1 vector (Shanghai enzyme research Co., Ltd.) to increase the mRNA and protein levels of FN1. Cells were cultured to $80 \%$ confluence in 6 -well plates, then either pcDNA3.1 vector $\left(3.0 \mu \mathrm{g}\right.$ per $1 \times 10^{4}$ cells/well $)$ or pcDNA3.1-FN1 were transfected into the cells using Lipofectamine 2000 (Invitrogen; Thermo Fisher Scientific, Inc.) according to the manufacturer's instructions. After transfection for $48 \mathrm{~h}$, the mRNA and protein levels of FN1 were detected by RT-qPCR and western blot analysis.

Grouping. JEG-3 or BeWo cells transfected with nonsense siRNA were identified as the siNC group; siRNA-FN1-transfected JEG-3 or BeWo cells were identified as the siFN1 group; pcDNA3.1 vector-transfected JEG-3 or BeWo cells were identified as the NC group; pcDNA3.1-FN1-transfected JEG-3 or BeWo cells were identified as the FN1 group. LY294002 (PI3K/Akt signaling pathway inhibitor) was purchased from MedChem Express and insulin-like growth factor-1 (IGF-1; PI3K/Akt signaling pathway activator) was purchased from PeproTech, Inc. After the JEG-3 cells were pre-treated with LY294002 $(5 \mu \mathrm{M})$ for $4 \mathrm{~h}$, pcDNA3.1-FN1 were then transfected into the JEG-3 cells (FN1 + LY294002). After the BeWo cells were pre-treated with IGF-1 $(5 \mu \mathrm{M})$ for $4 \mathrm{~h}$, siRNA-FN1 was then transfected into the BeWo cells (siFN1 + IGF-1).

Cell Counting kit $8(C C K-8)$ assay. The JEG-3 or BeWo cells in the logarithmic phase $(8,000$ cells/well $)$ were trypsinized and added into 96 -well plates for a 24 -h pre-incubation at $37^{\circ} \mathrm{C}$. Following incubation for $4 \mathrm{~h}$ at $37^{\circ} \mathrm{C}, \mathrm{CCK} 8$ solution $(10 \mu \mathrm{l})$ was injected to each well. The cells at 0 and $48 \mathrm{~h}$ were harvested using the CCK-8 kit (cat. no. C0037, Beyotime Institute of Biotechnology) according to the manufacturer's protocol. The absorption at different stages was recorded at $450 \mathrm{~nm}$ using a microplate reader (Thermo Fisher Scientific, Inc.).

Cell apoptosis assay. Cells in the different groups were cultured in a 6-well plate at a density of $2 \times 10^{4}$ cells/well for 
$24 \mathrm{~h}$. The cells were then centrifuged, collected and washed with PBS twice. The supernatant was discarded, and the cells were resuspended in $400 \mu \mathrm{l}$ of $1 \mathrm{X}$ Binding Buffer and incubated with $5 \mu \mathrm{l}$ of Annexin V-FITC for $15 \mathrm{~min}$ in the dark. Cells were mixed thoroughly with $10 \mu \mathrm{l}$ of PI staining solution (CA1020, Beijing Solarbio Science \& Technology Co., Ltd.) and incubated for $5 \mathrm{~min}$ in the dark. The proportion of cell apoptosis was assessed using a flow cytometer (CytoFLEX, Beckman Coulter, Inc.).

$R N A$ extraction and reverse transcription-quantitative PCR (RT-qPCR) assays. Total RNA was isolated from the pcDNA3.1-FN1-transfected JEG-3 and BeWo cells and siRNA-FN1-transfected JEG-3 and BeWo cells using TRIzol reagent (Invitrogen; Thermo Fisher Scientific, Inc.) following the manufacturer's instructions. Total RNA was quantified using a NanoDrop ND-100 Spectrophotometer (NanoDrop Technologies; Thermo Fisher Scientific, Inc.) at $260 \mathrm{~nm}$. Total RNA (2 $\mu \mathrm{g})$ was reverse transcribed into cDNA using SuperScript III (Invitrogen; Thermo Fisher Scientific, Inc.) according to the manufacturer's instructions. qPCR was performed using Fast SYBR Green Master Mix (Applied Biosystems) with an ABI PRISM7900 Sequence Detection System. The primers used for amplification were as follows: FN1 sense, 5'-TGGTATTCAGCTTCCTGGCA-3' and antisense, 5'-CGGGTATGGTCTTGGCCTAT-3'; and GAPDH sense, 5'-ACCCAGAAGACTGTGGATGG-3' and antisense, 5'-TCAGCTCAGGGATGACCTTG-3'. qPCR reaction included an initial denaturation step at $95^{\circ} \mathrm{C}$ for $10 \mathrm{~min}$, followed by 40 cycles at $95^{\circ} \mathrm{C}$ for $15 \mathrm{sec}$ and $60^{\circ} \mathrm{C}$ for $60 \mathrm{sec}$, and at $75^{\circ} \mathrm{C}$ to $95^{\circ} \mathrm{C}$; the temperature rises at $1^{\circ} \mathrm{C}$ per $20 \mathrm{sec}$. The relative mRNA expression was normalized to GAPDH, which was calculated based on the $\mathrm{Cq}$ value according to the equation: $2^{-\Delta \Delta \mathrm{Cq}}(22)$. Each sample was analyzed in triplicate.

Western blot analysis. Total proteins were extracted from the cells in the different groups using RIPA lysis buffer (cat. no. P0013B; Beyotime Institute of Biotechnology), according to the manufacturer's instructions. The protein concentration was measured using a BCA kit (Beyotime). A total of $10 \mu \mathrm{g}$ each sample was separated on a $10 \%$ SDS-PAGE, transferred to PVDF membranes (EMD Millipore) and then immunoblotted with antibodies against FN1 (1:800 dilution; ab2413, Abcam), cleaved caspase-3 (1:1,000 dilution; ab2302, Abcam), Bax (1:500 dilution; ab32503, Abcam), Bcl-2 (1:800 dilution; ab32124, Abcam), proliferating cell nuclear antigen (PCNA; 1:1,000 dilution; ab92552, Abcam), Ki67 (1:1,000 dilution; ab92742, Abcam), phosphorylated (p)-PI3K (1:500, abs130868, Absin Bioscience Inc.), PI3K (1:1,000, abs119725, Absin Bioscience, Inc.), p-Akt (1:800 dilution; ab38449, Abcam), Akt (1:800 dilution; ab8805, Abcam) and GAPDH (1:2,000 dilution; ab181602, Abcam), followed by incubation with HRP goat anti-rabbit IgG (AS014; ABclonal). As for FN1, cleaved caspase-3, Bax, Bcl-2, PCNA and Ki67 the dilution of the HRP goat anti-rabbit IgG antibody was 1:2,000; as for GAPDH, the dilution was 1:10,000. The primary antibodies FN1, cleaved caspase-3, Bax, Bcl-2, PCNA, Ki67 and GAPDH were incubated with the membranes at $4^{\circ} \mathrm{C}$ overnight. The secondary antibody HRP goat anti-rabbit $\mathrm{IgG}$ was incubated with the membranes at $37^{\circ} \mathrm{C}$ for $2 \mathrm{~h}$. The results were visualized using the Image Lab Imaging System software v4.0 (Bio-Rad Laboratories, Inc.).

Statistical analysis. The mean value of FN1 expression in the SA group of $>2.43$ was defined as a high expression. The associations of FN1 expression with the clinicopathological features of the patients were examined using the $\chi^{2}$ test. Each experiment was repeated at least 3 times. The statistical analyses of the experimental data were performed using a two-tailed Student's paired t-test and one-way ANOVA followed by Tukey's post hoc test. Statistical significance was assessed at least three independent experiments and a P-value $<0.05$ was considered to indicate a statistically significant difference.

\section{Results}

DEGs and hub genes identified in gene set 1 of the GSE127170 dataset. The GSE127170 dataset was downloaded from the GEO database. The analysis of the dataset revealed 903 differentially expressed genes (DEGs) $(410 \mathrm{P}<0.05$ and $|\log F C|>1)$, including 493 upregulated and 410 downregulated genes (Fig. 1A). The top 300 DEGs were used to construct a PPI network using the STRING website, which was visualized using Cytoscape (Fig. 1B). The top 20 hub genes were identified by the MCC and Degree method using the cytoHubba plug-in of Cytoscape (Fig. 1C and D). As shown in Fig. 1E, a total of 17 overlapping hub genes were identified: LYN proto-oncogene, scr family tyrosine kinase (LYN), Wnt family member 3A (WNT3A), Fos proto-oncogene, AP-1 transcription factor subunit (FOS), transforming growth factor beta 1 (TGFB1), mitogen-activated protein kinase 8 (MAPK8), CD68 molecule (CD68), insulin like growth factor 1 receptor (IGF1R), glutamate ionotropic receptor nmDA type subunit 2B (GRIN2B), nuclear receptor subfamily 4 group A member 1 (NR4A1), vascular endothelial growth factor B (VEGFB), Kruppel like factor 4 (KLF4), TIAM Rac1 associated GEF 1 (TIAM1), nitric oxide synthase 3 (NOS3), FYN proto-oncogene, Src family tyrosine kinase (FYN), secreted frizzled related protein 1 (SFRP1), AXIN2 and FN1.

DEGs and hub genes identified in gene set 2 of the GSE127170 dataset. The GSE127170 dataset was downloaded from the GEO database. The analysis of the dataset revealed 440 differential genes (DEGs), including 260 upregulated and 180 downregulated genes (Fig. 2A). The top 300 DEGs were used to construct a PPI network using the STRING website, which was visualized using Cytoscape (Fig. 2B). The top 20 hub genes were identified by the MCC and Degree methods using the cytoHubba plug-in of Cytoscape (Fig. 2C and D). As shown in Fig. 2E, a total of 16 overlapping hub genes were identified: Yes associated protein 1 (YAP1), integrin subunit alpha 1 (ITGA1), colony stimulating factor 1 receptor (CSF1R), CD9 molecule (CD9), melanoma cell adhesion molecule (MCAM), integrin subunit alpha M (ITGAM), collagen type I alpha 1 chain (COL1A1), TGFB1, protein kinase cAMP-activated catalytic subunit beta (PRKACB), interferon regulatory factor 8 (IRF8), annexin A1 (ANXA1), transgelin (TAGLN), KLF4, FYN, collagen type IV alpha 1 chain (COL4A1) and FN1. 

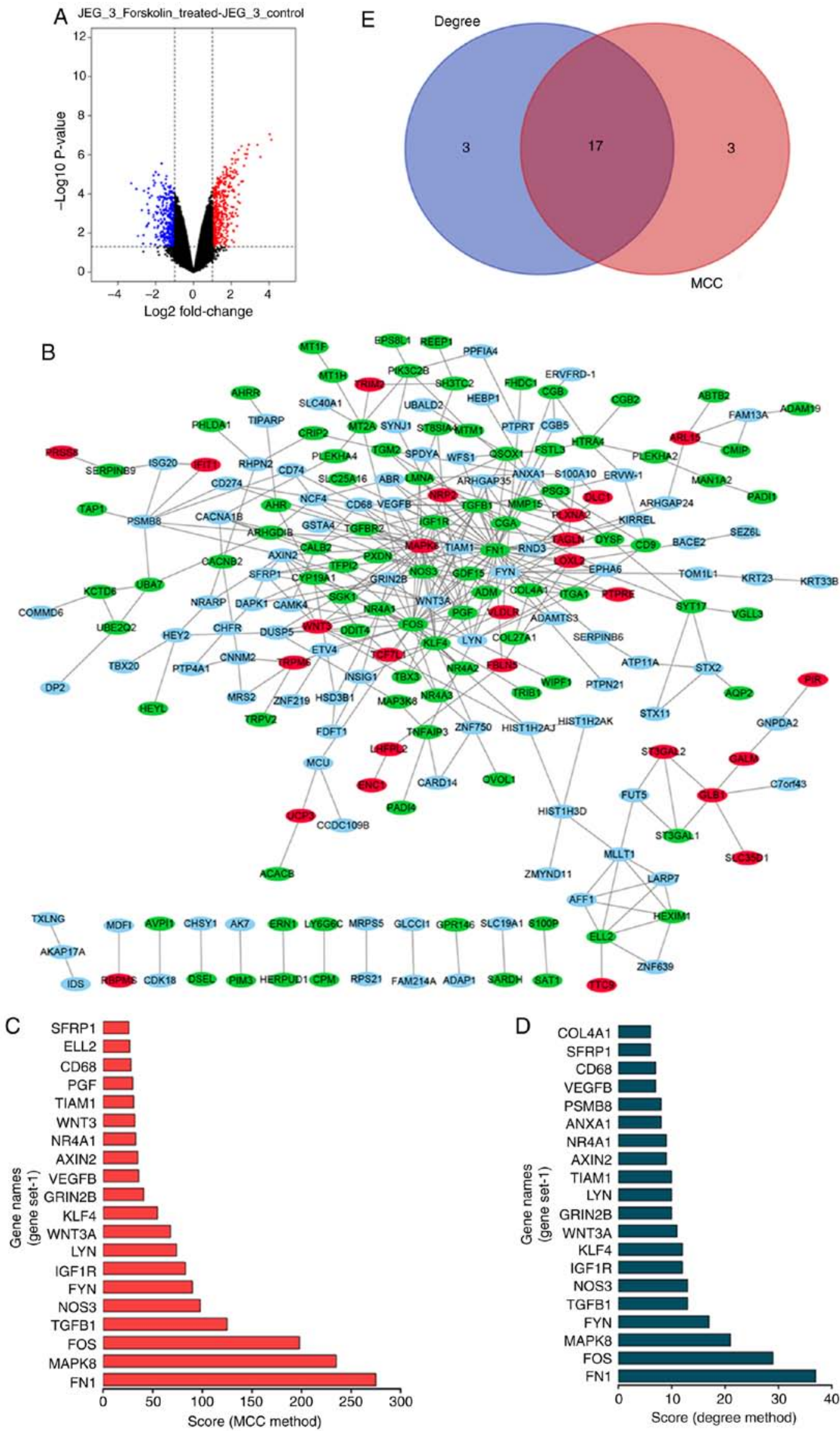

Figure 1. Identification of hub genes and PPI network in gene set1 from the GSE127170 dataset. (A) Volcano plot of 493 genes was significantly up-regulated and Volcano plot of 410 genes was significantly downregulated, $\log \mathrm{FC}>-1$ or $<1$ and $\mathrm{P}<0.05$; (B) top 300 DEGs were used to construct the PPI network (red denotes downregulation and green denotes upregulation; (C) hub genes were identified by using the plug-in cytoHubba from Cytoscape software according to MCC method; (D) hub genes were identified by using the plug-in cytoHubba from Cytoscape software according to the Degree method; (E) Venn diagram of 17 overlapping hub genes. 
A

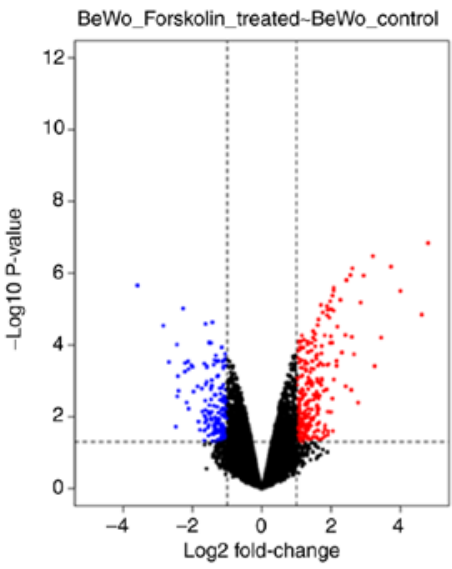

$\mathrm{E}$

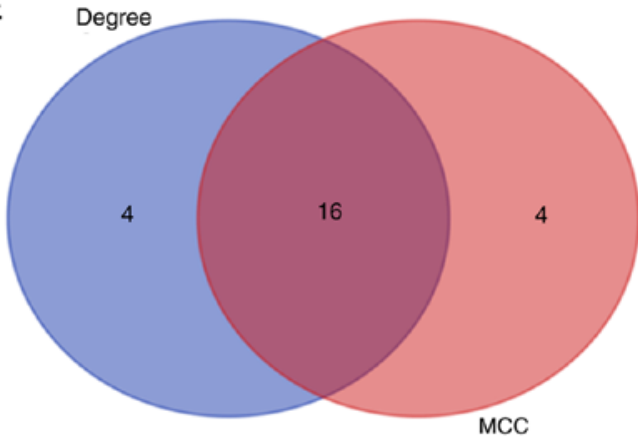

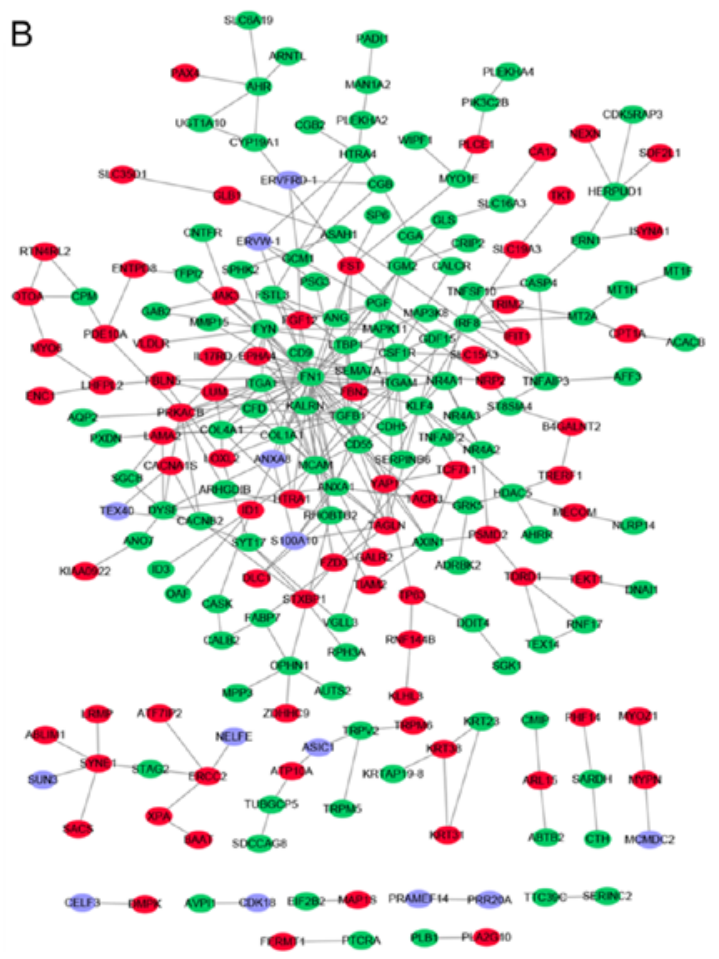
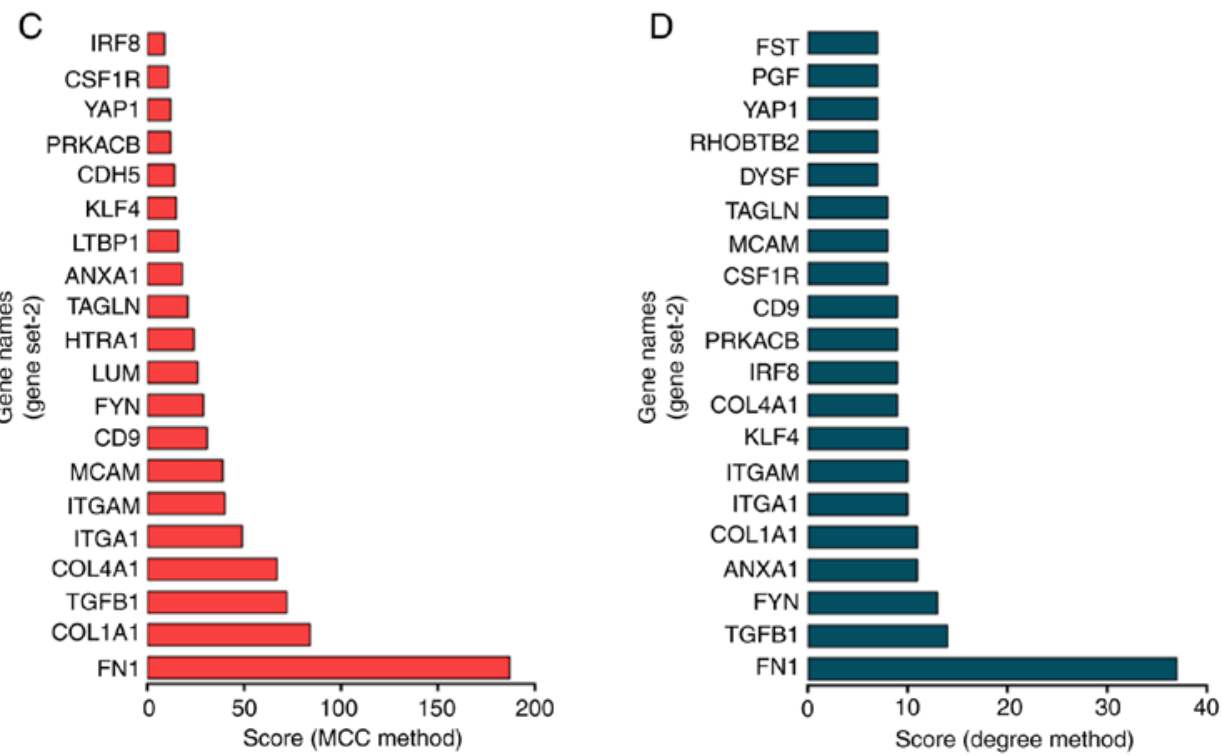

Figure 2. Identification of hub genes and PPI network in gene set2 from the GSE127170 dataset. (A) Volcano plot of 260 genes was significantly upregulated and Volcano plot of 180 genes was significantly downregulated, $\log F C>-1$ or $<1$ and $\mathrm{P}<0.05$; (B) top 300 DEGs were used to construct the PPI network (red denotes downregulation and green denotes upregulation; (C) hub genes were identified by using the plug-in cytoHubba from Cytoscape software according to MCC method; (D) hub genes were identified by using the plug-in cytoHubba from Cytoscape software according to the Degree method; (E) Venn diagram of 16 overlapping hub genes. 

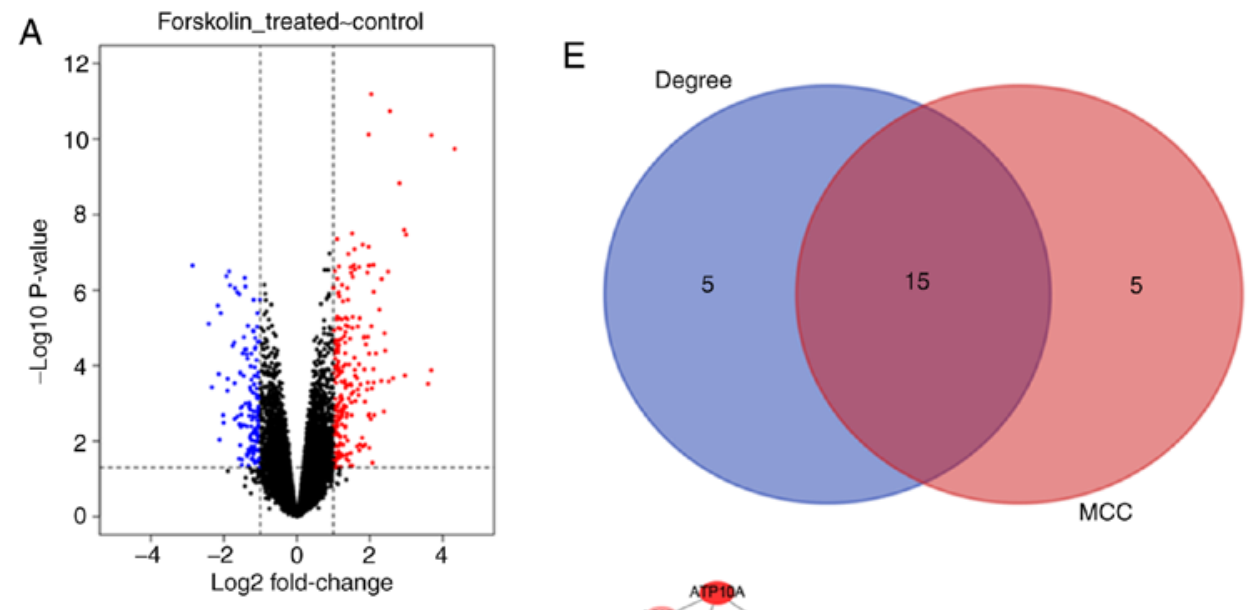

B
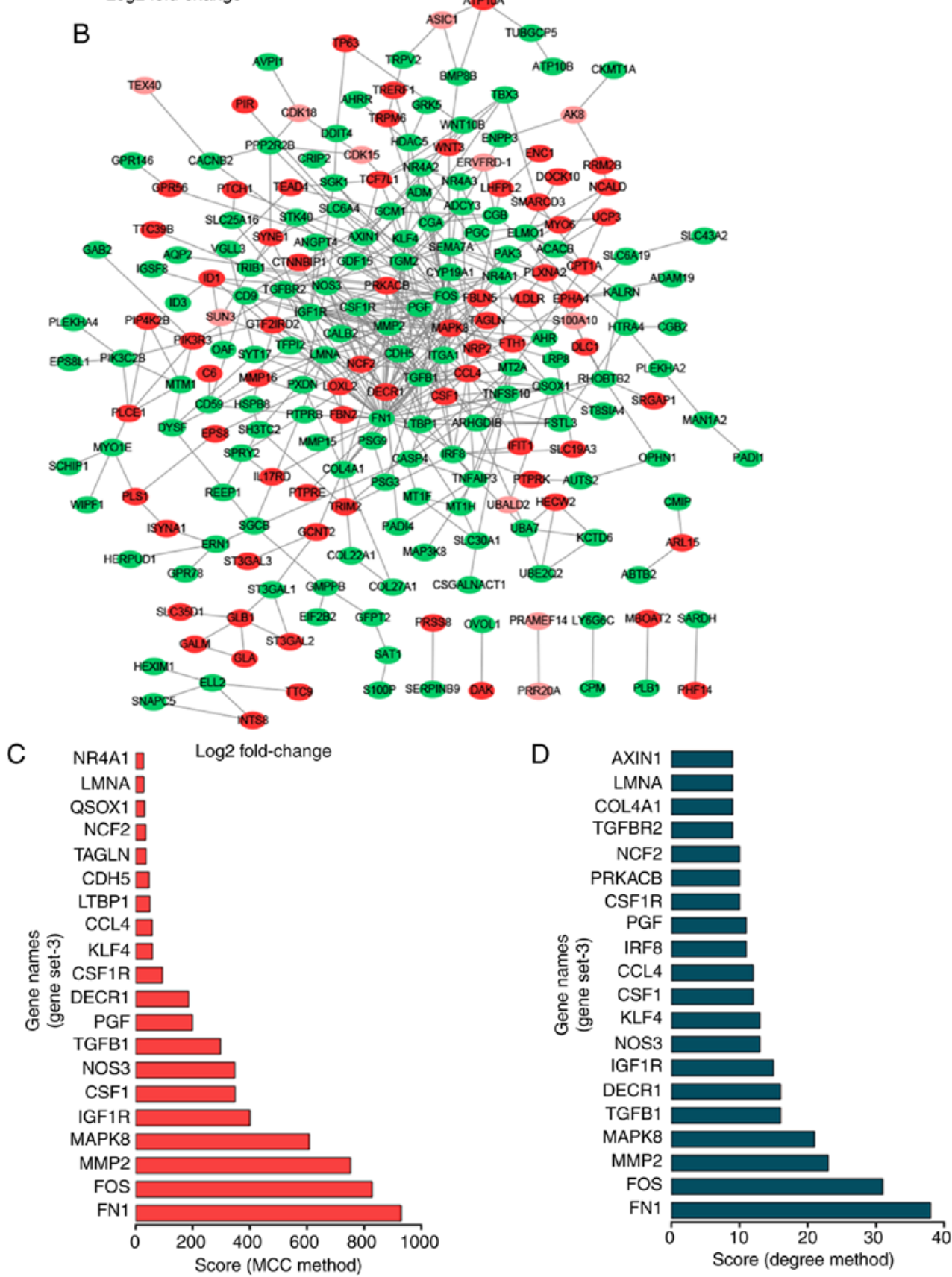

Figure 3. Identification of hub genes and PPI network in gene set3 from the GSE127170 dataset. (A) Volcano plot of 238 genes was significantly upregulated and Volcano plot of 150 genes was significantly downregulated, $\log \mathrm{FC}>-1$ or $<1$ and $\mathrm{P}<0.05$; (B) top 300 DEGs were used to construct the PPI network (red denotes downregulation and green denotes upregulation; (C) hub genes were identified by using the plug-in cytoHubba from Cytoscape software according to the MCC method; (D) hub genes were identified by using the plug-in cytoHubba from Cytoscape software according to the Degree method; (E) Venn diagram of 15 overlapping hub genes. 
A

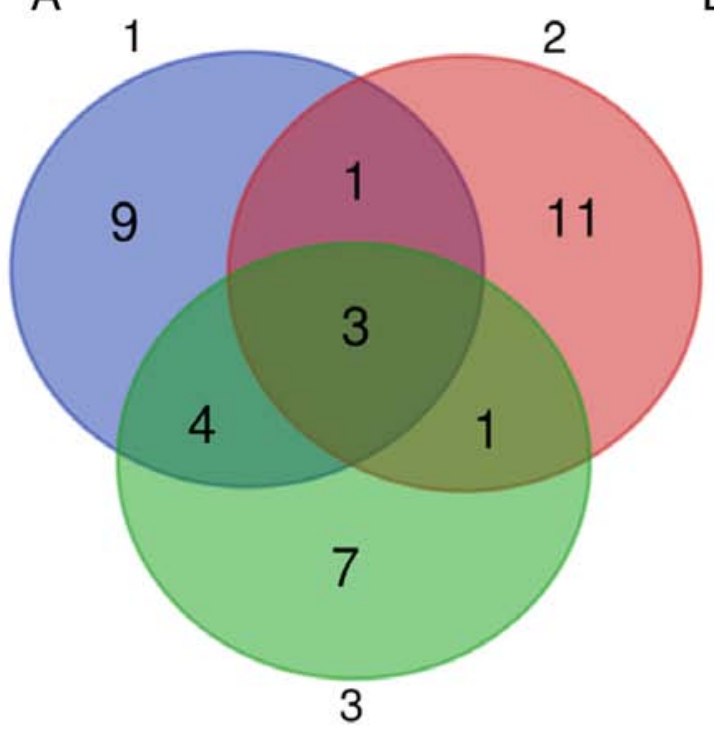

B

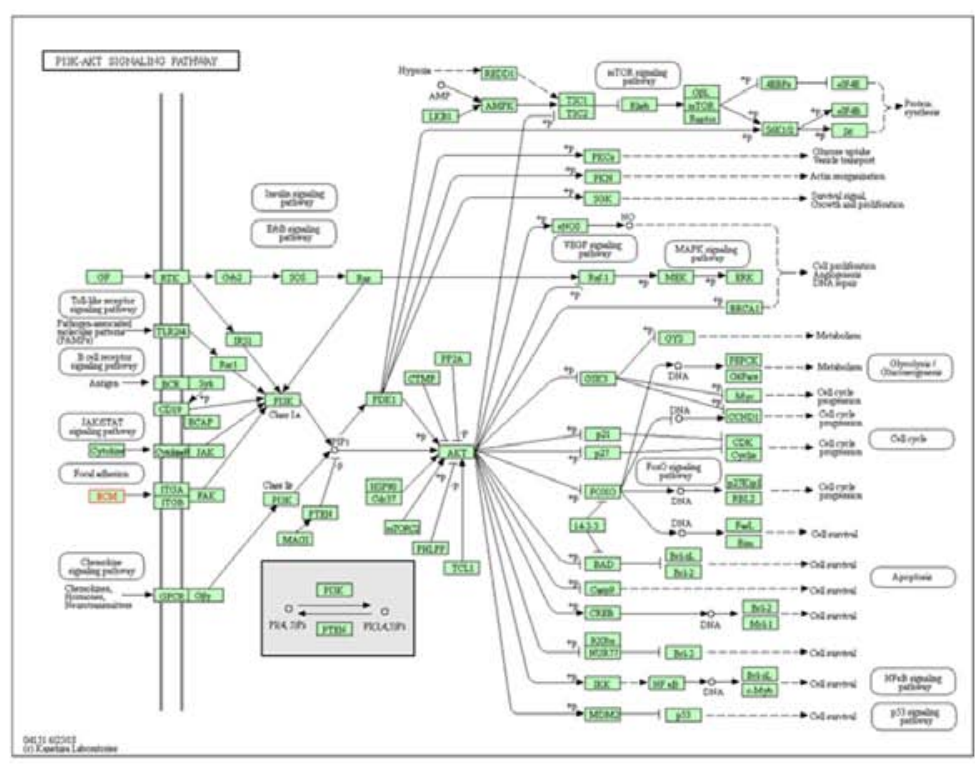

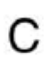

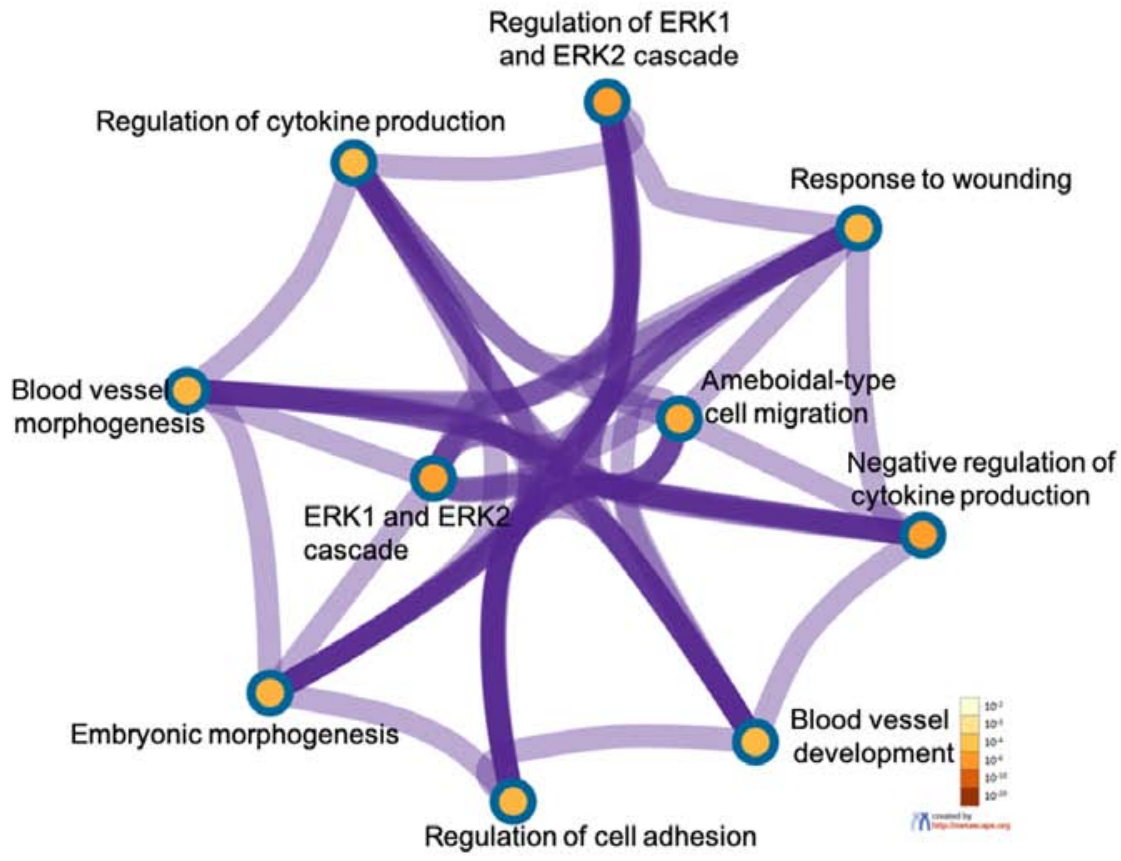

Figure 4. GO and KEGG analysis of hub genes. (A) Venn diagram of 3 overlapping genes from hub genes of gene set1, gene set2 and gene set3; (B) KEGG enrichment analysis revealed that FN1 was mainly enriched in the PI3K/Akt signaling pathway; (C) GO enrichment analysis revealed that the overlapping genes mainly were enriched in the biological process (BP) term. FN1, fibronectin 1.

DEGs and hub genes identified in gene set 3 of the GSE127170 dataset. The GSE127170 dataset was downloaded from the GEO database. The analysis of the dataset revealed 388 differentially expressed genes (DEGs), including 238 upregulated and 150 downregulated genes (Fig. 3A). The top 300 DEGs were used to construct a PPI network using the STRING website, which was visualized in Cytoscape (Fig. 3B). The top 20 hub genes were identified by the MCC and Degree methods using the cytoHubba plug-in of Cytoscape (Fig. 3C and D). As shown in Fig. 3E, a total of 15 overlapping hub genes were identified: Placental growth factor (PGF), colony stimulating factor 1 receptor (CSF1R), matrix metallopeptidase 2(MMP2), amin A/C (LMNA), FOS, TGFB1, C-C motif chemokine ligand 4 (CCL4), MAPK8, IGF1R, colony stimulating factor 1
(CSF1), KLF4, nitric oxide synthase 3 (NOS3), neutrophil cytosolic factor 2 (NCF2), 2,4-dienoyl-CoA reductase 1 (DECR1) and FN1.

GO and KEGG enrichment analysis. TGFB1, FN1 and KLF4 were overlapping hub genes from gene set1, gene set 2 and gene set3 (Fig. 4A). FN1 was mainly enriched in the PI3K/Akt signaling pathway (Fig. 4B). TGFB1, FN1 and KLF4 were mainly enriched in the biological process (BP) term, including the regulation of the ERK1 and ERK2 cascade, the response to wounding, ameboidal-type cell migration, the negative regulation of cytokine production, blood vessel development, the regulation of cell adhesion, embryonic morphogenesis, the ERK1 and ERE2 cascade, blood vessel morphogenesis and the 
A

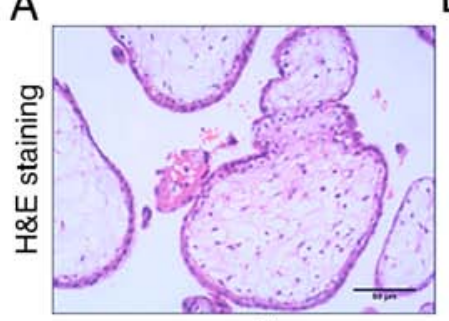

IA

E

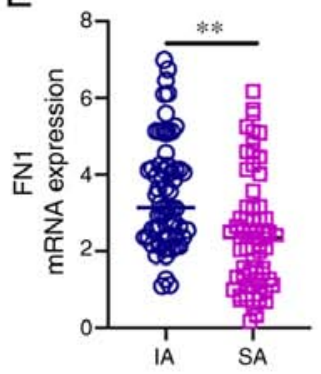

$\mathrm{F}$
B

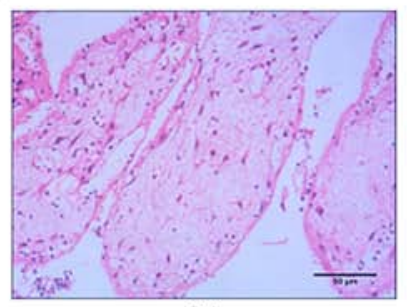

SA
C

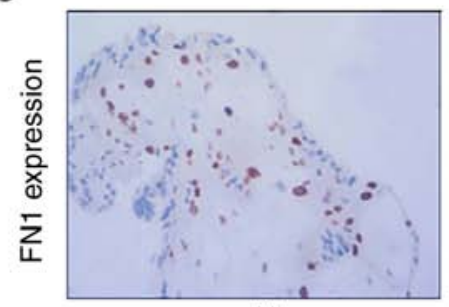

IA
D

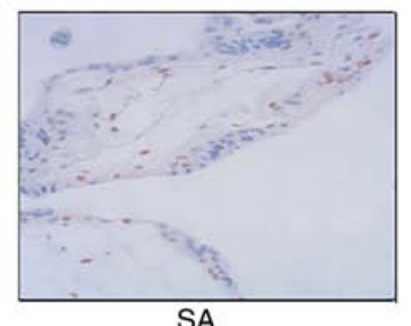

SA

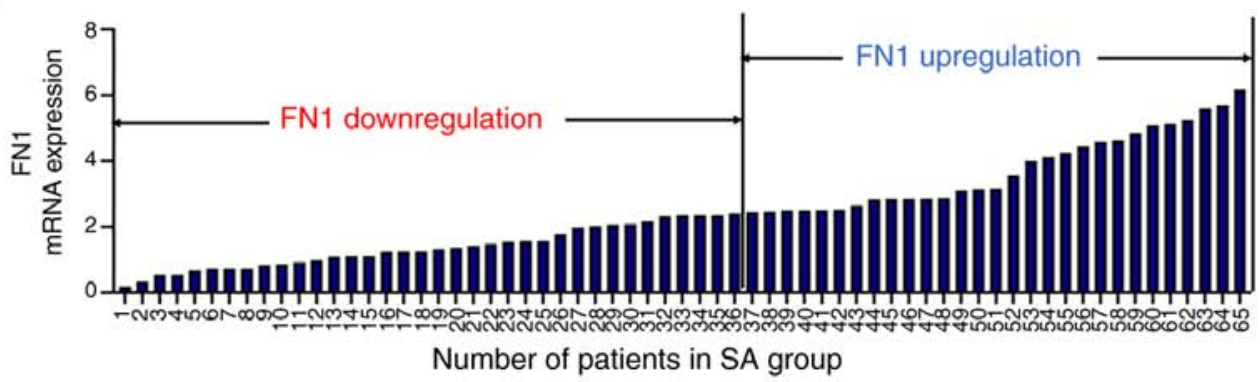

Figure 5. FN1 expression is downregulated in the chorionic villus tissues obtained from placenta of patients diagnosed with spontaneous abortion. (A) H\&E staining was used to examine the pathological features of chorionic villus tissues obtained from placentas in the IA group; (B) H\&E staining was used to investigate the pathological features of chorionic villus tissues obtained from placentas in the SA group. (C) FN1 expression of the chorionic villus tissues obtained from placentas was measured by immunohistochemistry in the IA group. (D) FN1 expression of the chorionic villus tissues obtained from placentas was measured by immunohistochemistry in the SA group. (E) FN1 mRNA level between IA group and SA group was measured by RT-qPCR. (F) FN1 mRNA level in the SA group was measured by RT-qPCR. GAPDH was used as a load control. Data are presented as the means \pm standard deviation. ${ }^{* *} \mathrm{P}<0.01$ vs. IA group. FN1, fibronectin 1; SA, spontaneous abortion; IA, induced abortion.

regulation of cytokine production using the Metascape website (Fig. 4C).

FN1 expression is downregulated in chorionic villus tissues from the placentas of patients diagnosed with SA. No change was observed in the morphology of the chorionic villus tissues of patients in the IA group. The cytotrophoblasts in the inner layer were cubic, polygonal, or oval, with clear boundaries and a transparent cytoplasm. The outer syncytiotrophoblasts contained multiple nuclei. Stellate mesenchymal cells, myxoid matrix and round or oval cells were observed in the chorionic villus tissue and there were no blood vessels in the chorionic villus tissue (Fig. 5A). By contrast, the morphology of chorionic villus tissues in the SA group were significantly altered. Microscopic examination revealed different degrees of degenerative changes, proliferation and degeneration in the chorionic villus tissues. The number of cytotrophoblasts and syncytiotrophoblasts were significantly decreased or were absent. In addition, mesenchymal celluloid degeneration, structural destruction, homogeneous shaped cells, matrix fiber-like degeneration, and myxoid degeneration were observed. The basement membrane of the villous trophoblast cells and blood capillaries was slightly thickened (Fig. 5B). IHC revealed FN1 staining in the chorionic villi and surrounding tissues in the IA group (Fig. 5C). By contrast, the chorionic villi and surrounding tissues in the SA group exhibited only weak FN1 staining (Fig. 5D). The FN1 mRNA levels in the sera of patients in the SA group were significantly lower than that those in the IA group (Fig. 5E). FN1 expression was $<2.43$ in 36 patients in the SA group (Fig. 5F). The analysis of the association between FN1 expression and the patient characteristics revealed that FN1 was not associated with age $(\mathrm{P}=0.314)$ or pregnancy cycle $(\mathrm{P}=0.718$; Table $\mathrm{I})$.
FN1 regulates the viability and apoptosis of JEG-3 and BeWo cells by modulating the expression of proliferationand apoptosis-related proteins. JEG-3 and BeWo cells were transfected with siRNA-FN1 or pcDNA-FN1, and western blot analysis and RT-qPCR were then conducted to assess the FN1 levels. The results revealed that FN1 protein and mRNA levels were significantly decreased in the siFN1-transfected cells and were significantly increased in pcDNA-FN1-transfected cells (Fig. 6A and $\mathrm{B}$ ). The results of CCK- 8 assay revealed that the knockdown of FN1 prominently decreased the viability of the JEG-3 and BeWo cells, whereas the overexpression of FN1 notably increased cell viability (Fig. 6C). The results also revealed that FN1 knockdown promoted cell apoptosis, whereas FN1 overexpression inhibited the apoptosis of JEG-3 and BeWo cells (Fig. D and E). FN1 silencing resulted in increased protein levels of cleaved caspase- 3 and Bax, and decreased protein levels of Bcl-2. It also resulted in significantly decreased protein levels of PCNA and Ki67 in the JEG-3 and BeWo cells. However, FN1 overexpression markedly decreased cleaved caspase- 3 and Bax protein levels, and increased Bcl-2, PCNA and Ki67 protein levels in the JEG-3 and BeWo cells, as shown by western blot analysis (Fig. 6F and $\mathrm{G})$.

FN1 regulates the viability and the apoptosis of JEG-3 and BeWo cells by affecting the activation of the PI3K/Akt signaling pathway. The PI3K and Akt phosphorylation levels were significantly decreased by FN1 silencing and were notably increased by FN1 overexpression in the JEG-3 and BeWo cells (Fig. 7A and B). The results of CCK-8 assay revealed that the promoting effects of FN1 overexpression on the viability of the JEG-3 cells were blocked by the 

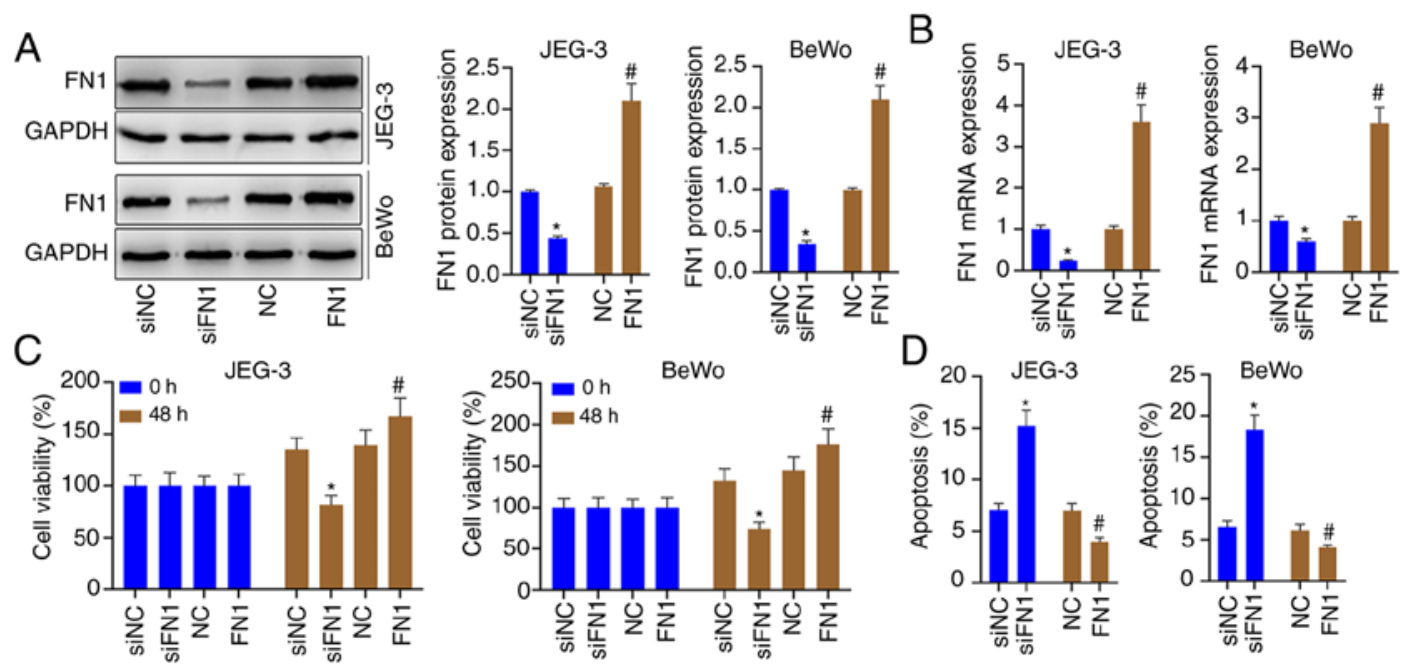

E
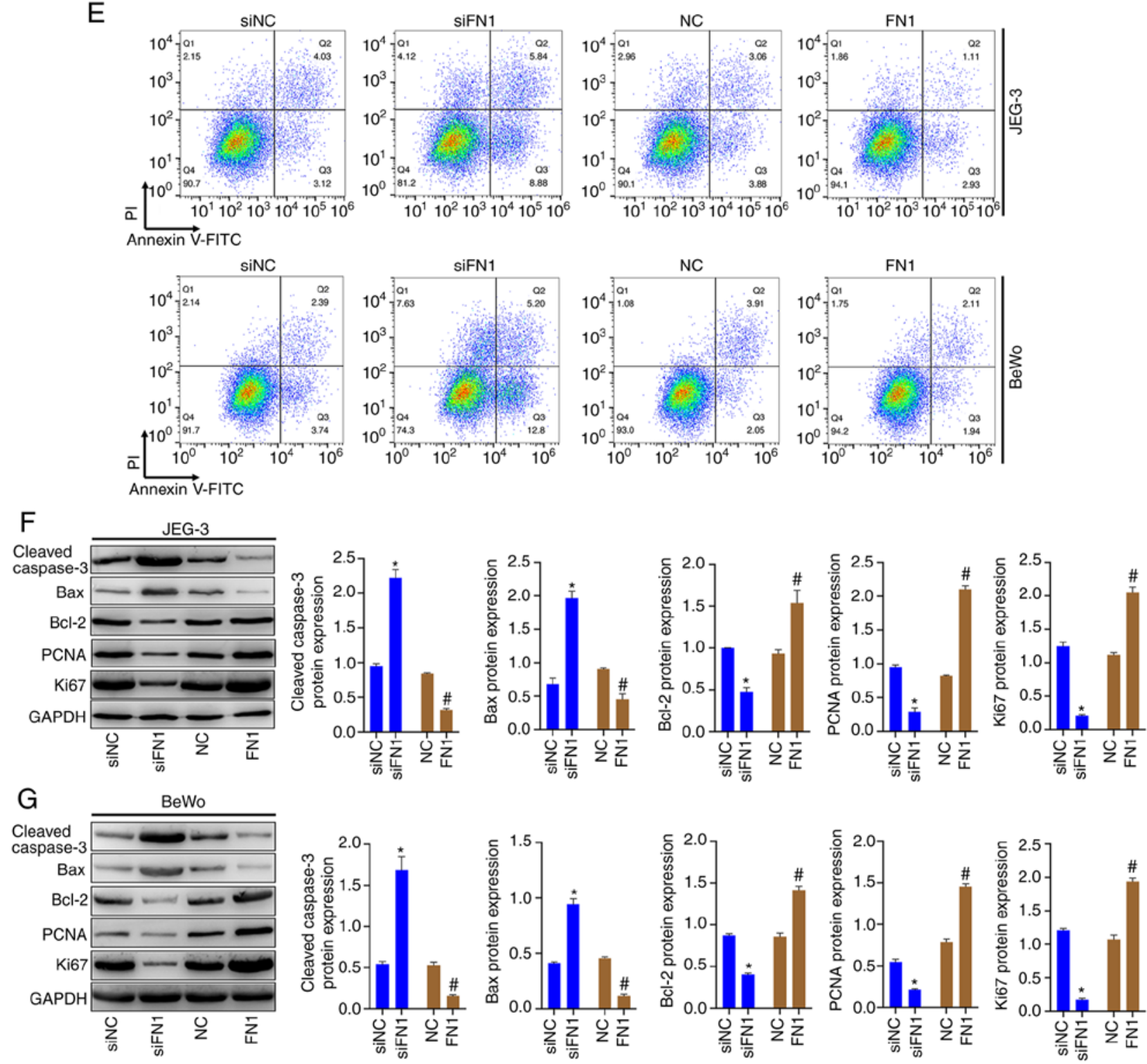

Figure 6. FN1 regulates the viability and apoptosis of JEG-3 and BeWo cells via regulating the proliferation and apoptosis-related protein expression. (A) Western blot analysis was used to determine the protein level of FN1 in siRNA-FN1 or pcDNA-FN1 transfected JEG-3 and BeWo cells. (B) RT-qPCR assay was used to determine the mRNA level of FN1 in siRNA-FN1- or pcDNA-FN1-transfected JEG-3 and BeWo cells. (C) CCK-8 assay was used to determine the viability of siRNA-FN1- or pcDNA-FN1-transfected JEG-3 and BeWo cells at 0 and $48 \mathrm{~h}$. (D) Graphs of the apoptosis of siRNA-FN1- or pcDNA-FN1-transfected JEG-3 and BeWo cells. (E) Flow cytometry was used to determine the apoptosis of siRNA-FN1- or pcDNA-FN1-transfected JEG-3 and BeWo cells. (F) Western blot analysis was used to determine the protein level of cleaved caspase-3, Bax, bcl-2, PCNA and Ki67 in siRNA-FN1- or pcDNA-FN1-transfected JEG-3 cells. (G) Western blot analysis was used to determine the protein level of cleaved caspase-3, Bax, bcl-2, PCNA and Ki67 in siRNA-FN1- or pcDNA-FN1-transfected BeWo cells. GAPDH was used as a loading control. Data are presented as the means \pm standard deviation. ${ }^{*} \mathrm{P}<0.05$ vs. siNC group and ${ }^{\#} \mathrm{P}<0.05$ vs. NC group. FN1, fibronectin 1 . 

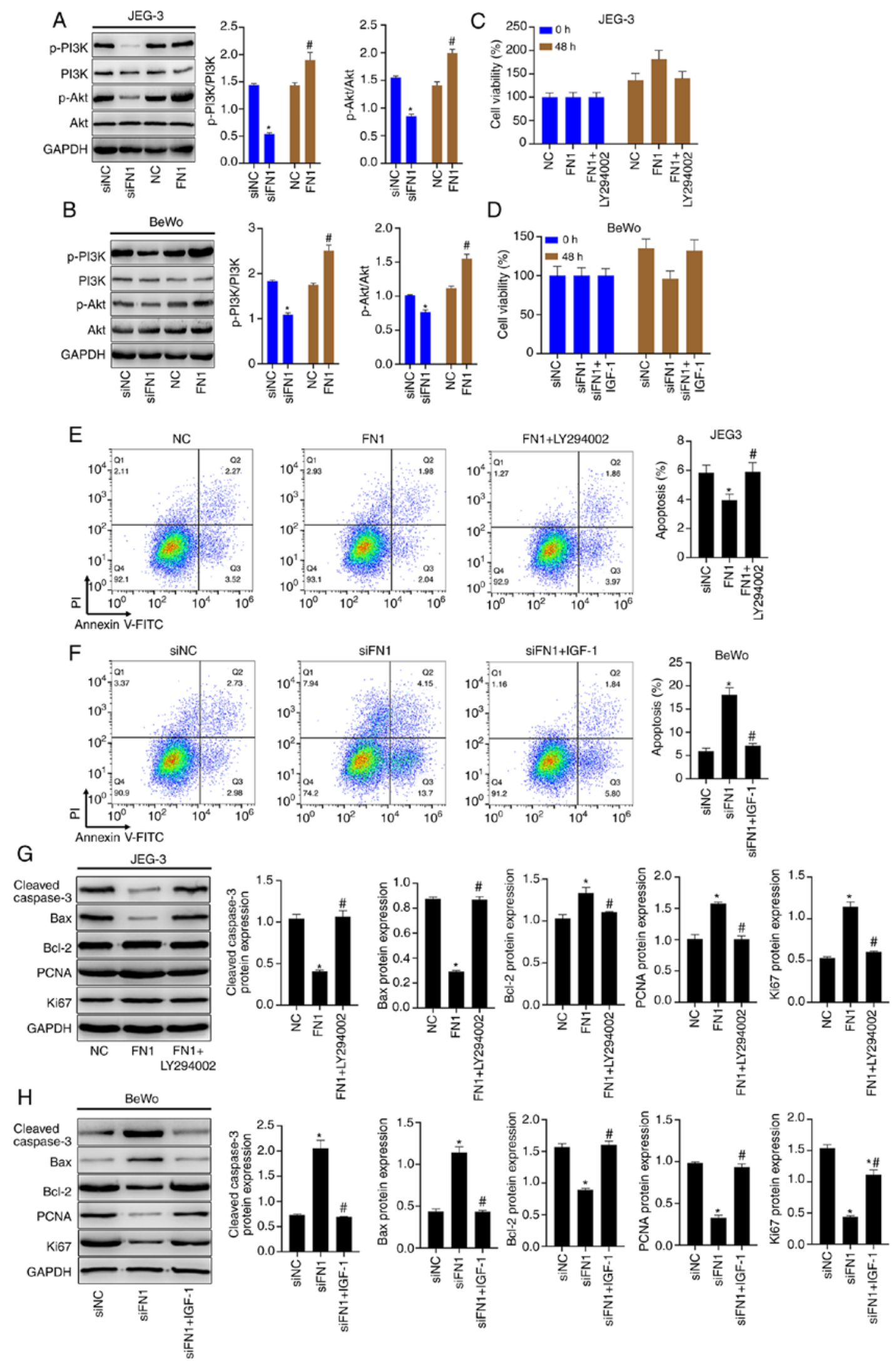

Figure 7. FN1 regulates the viability and apoptosis of JEG-3 and BeWo cells by affecting the activation of the PI3K/Akt signaling pathway. (A) Western blot analysis was used to determine the phosphorylation level of PI3K and Akt siRNA-FN1- or pcDNA-FN1-transfected JEG-3 cells. (B) Western blot analysis was used to determine the phosphorylation level of PI3K and Akt siRNA-FN1- or pcDNA-FN1-transfected BeWo cells. (C) CCK-8 assay was used to determine the viability of LY294002-treated JEG-3 cells transfected with pcDNA-FN1. (D) CCK-8 assay was used to determine the viability of IGF-1-treated BeWo cells transfected with siRNA-FN1. (E) Flow cytometry was used to determine the apoptosis of LY294002-treated JEG-3 cells transfected with pcDNA-FN1. (F) Flow cytometry was used to determine the apoptosis of IGF-1-treated BeWo cells transfected with siRNA-FN1. (G) Western blot analysis was used to determine the protein level of cleaved caspase-3, Bax, Bcl-2, PCNA and Ki67 in LY294002-treated JEG-3 cells transfected with pcDNA-FN1. (H) Western blot analysis was used to determine the protein level of cleaved caspase-3, Bax, Bcl-2, PCNA and Ki67 in IGF-1-treated BeWo cells transfected with siRNA-FN1. GAPDH was used as a loading control. Data are presented as the means \pm standard deviation. ${ }^{*} \mathrm{P}<0.05$ vs. siNC/NC group and ${ }^{\#} \mathrm{P}<0.05$ vs. FN1/siFN1 group. FN1, fibronectin 1 . 
A

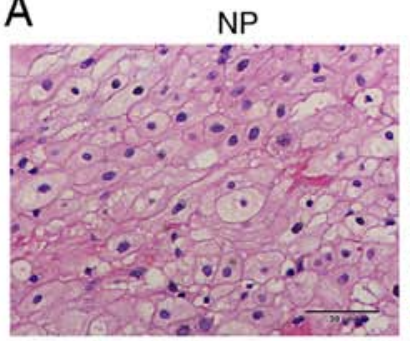

$$
\mathrm{FN}^{-1-}
$$
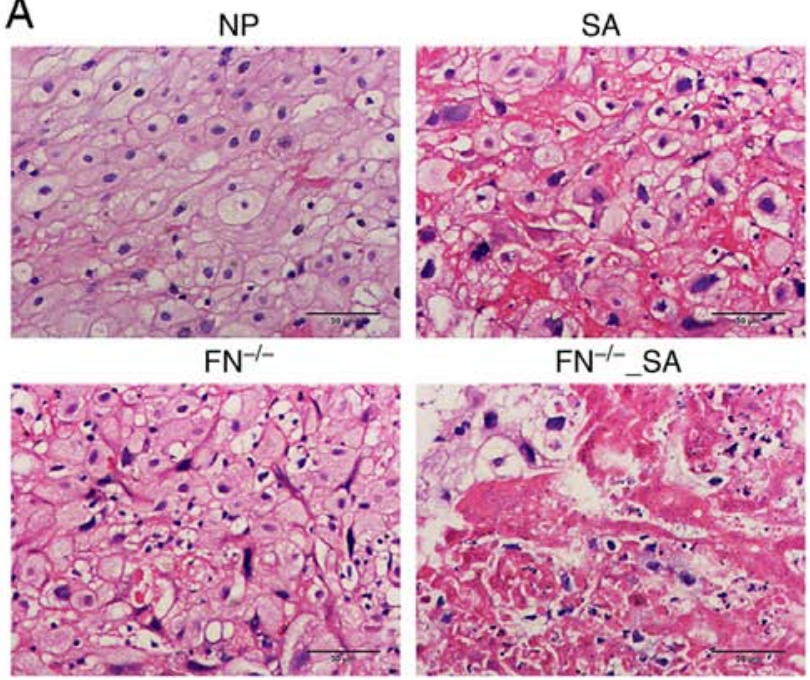

$\mathrm{FN}^{-1-} \_\mathrm{SA}$

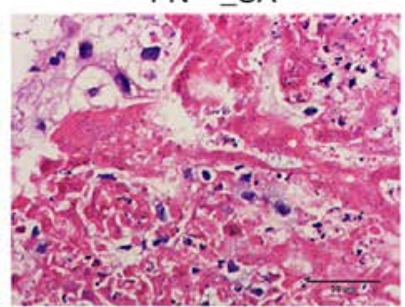

C

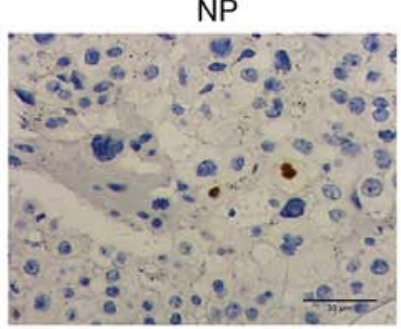

$\mathrm{FN}^{-1-}$

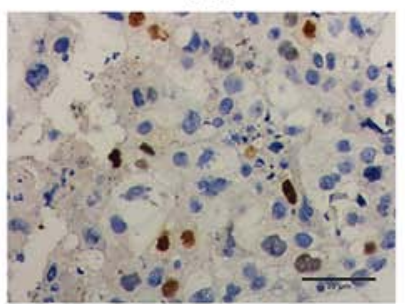

SA

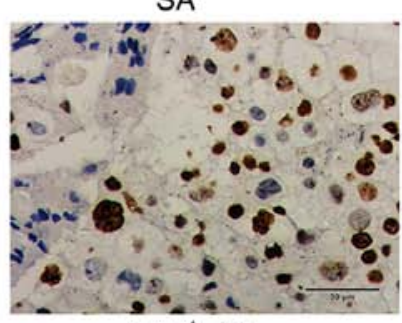

$\mathrm{FN}^{-1-} \mathrm{SA}$

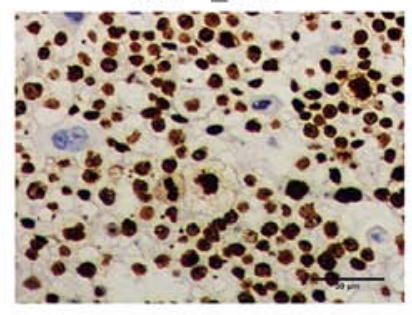

\section{B}
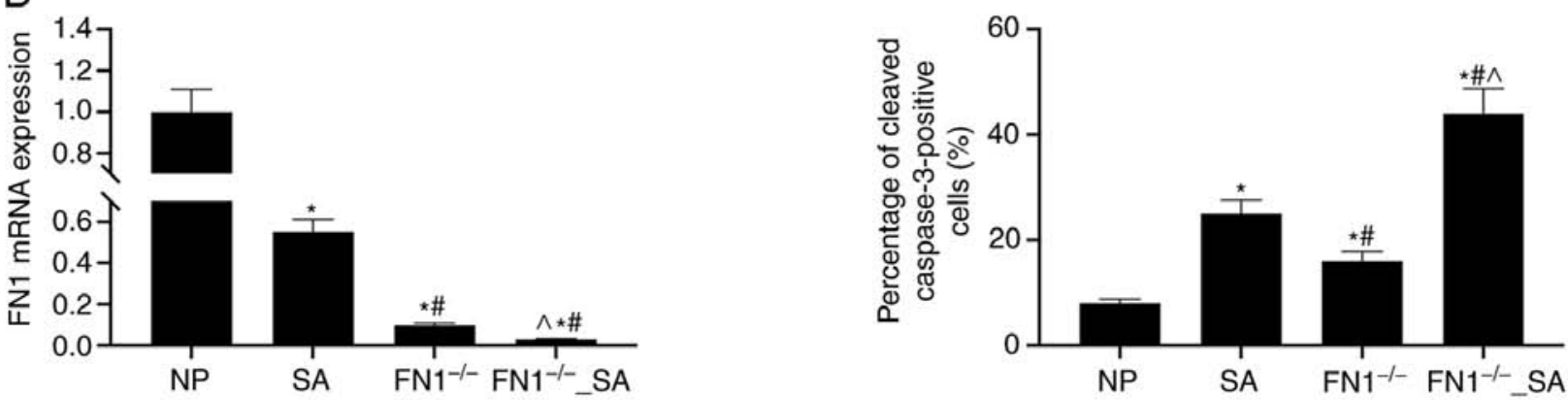

Figure 8. FN1 knockdown aggravates the apoptosis of trophoblasts in the chorionic villus tissues obtained from mice subjected to SA. (A) H\&E staining was used to detect histopathological changes in the chorionic villus tissues of mice in the NP, SA, FN1 $1^{-1}$, and FN1 ${ }^{-/}$_SA groups. (B) RT-qPCR was used to determine the mRNA level of FN1 in chorionic villus tissues of mice in the NP, SA, FN1 $1^{-/}$and $\mathrm{FN}^{-/}$_SA groups. (C) IHC assay was used to detect FN1 expression in chorionic villus tissues of mice in the NP, $\mathrm{SA}, \mathrm{FN1}^{--}$and $\mathrm{FN1}^{-{ }_{-}}$SSA groups. GAPDH was used as a loading control. Data are presented as the means \pm standard deviation. " $\mathrm{P}<0.05$ vs. NP group; ${ }^{\sharp} \mathrm{P}<0.05$ vs. SA group; and ${ }^{\wedge} \mathrm{P}<0.05$ vs. $\mathrm{FN1}{ }^{-/}$group. FN1, fibronectin 1 ; $\mathrm{SA}$, spontaneous abortion; NP, normal pregnancy.

PI3K/Akt signaling pathway inhibitor, LY294002 (Fig. 7C), and the inhibitory effects of FN1 silencing on BeWo cell viability were reversed by the PI3K/Akt signaling pathway activator, IGF-1 (Fig. 7D). LY294002 significantly promoted the apoptosis of FN1-overexpressing JEG-3 cells (Fig. 7E), and IGF-1 significantly blocked the induction of apoptosis of the FN1-silenced BeWo cells (Fig. 7F). In addition, the FN1 overexpression-mediated decrease in the levels of cleaved caspase- 3 and Bax, and the increase in the protein levels of Bcl-2, PCNA and Ki67 in the JEG-3 cells were reversed by LY294002 (Fig. 7G). The positive effects of FN1 inhibition on cleaved caspase- 3 and Bax protein levels, and the negative effects on Bcl-2, PCNA and Ki67 protein levels in the BeWo cells were reversed by IGF-1 (Fig. 7H).

FN1 knockout increases the apoptosis of trophoblasts in chorionic villus tissues from mice subjected to SA. The histopathological changes in the chorionic villus tissues of mice in the $\mathrm{NP}, \mathrm{FN}^{-/}, \mathrm{SA}$, and $\mathrm{FN}^{-/} \_\mathrm{SA}$ groups were observed by $\mathrm{H} \& \mathrm{E}$ staining. The organization of the chorionic villus tissues of mice in the NP group were normal, and the cell arrangement was tight. However, there were more histopathological changes in the chorionic villus tissues of mice in the $\mathrm{FN}^{-/}$group than in those of the NP group, but less than in those of the SA and $\mathrm{FN}^{-/-}$-SA groups (Fig. 8A). In addition, a morphological examination revealed that the pathological changes in the chorionic villus tissues of mice in the $\mathrm{FN}^{-/-}-\mathrm{SA}$ group were greater than the changes observed in the SA group. A comparison of the FN1 expression levels in the chorionic villus tissues from the mice in these groups revealed a gradual decrease across the NP, SA, $\mathrm{FN}^{-/-}$, and $\mathrm{FN}^{-/-}$_SA groups (Fig. 8B). SA or FN1 knockout alone increased the levels of cleaved caspase-3 in chorionic villus tissues, and FN1 knockout further increased the cleaved caspase- 3 levels in the chorionic villus tissues of mice subjected to SA (Fig. 8C).

\section{Discussion}

Pregnancy is a complex biological process. The successful implantation of the embryo is closely related to the development of the blastocyst, the growth of trophoblasts, and the connection between the maternal-fetal interface and immune regulation (23). Trophoblasts are a specific type of placental cells that play important roles in embryo implantation and the formation of the maternal-fetal interface (1). Apoptosis is a necessary physiological process for uterine decidual reconstruction, trophoblast invasion of the luteus and maintaining the immune tolerance balance at the maternal-fetal interface (5). Animal studies have indicated that compared with normal pregnancy CBA/JxBALB/c model mice, the SA 
CBA/JxDBA/2 model mice exhibit greater apoptosis in the villi and decidual tissues, and the apoptotic index is significantly higher than that of normal pregnancy model mice $(24,25)$. The excessive apoptosis of trophoblasts leads to intracellular dysfunction and abortion. The present bioinformatics analysis of the GSE127170 dataset revealed that TGFB1, FN1 and KLF4 are hub genes.

It has been reported that the disruption of the binding between TGFB1 and its receptor, TGFBR, can affect TGFB1 signal transduction, thus blocking nitric oxide synthase activity, leading to a decrease in nitric oxide synthesis, an increase in blood pressure and thrombosis, maternal hypoxia, an insufficient nutrient supply, and neovascularization (26-28). It has also been reported that FN1 is highly expressed in chorionic leukocytes and is related to the invasion, proliferation and adhesion of trophoblasts in the uterus $(29,30)$. In addition, FN1 was the top hub gene in 3 PPI networks generated using the MCC and Degree methods. KLF4 is considered to be a tumor suppressor gene, and its overexpression can promote the apoptosis of endometrial cancer cells (31). GO and KEGG analysis revealed that FN1 mainly affects the PI3K/Akt signaling pathway and is involved in cell proliferation and apoptosis (Fig. 4). Therefore, the effects of FN1 on trophoblasts has become the primary focus of our research group.

The results of the present study revealed that the morphology of the chorionic villi in the SA group differed significantly from that in the IA group. FN1 mRNA and protein levels were significantly higher in the chorionic villus and surrounding tissues in the IA group than in the SA group. In addition, the FN1 mRNA level was not significantly associated with the age of the pregnancy cycle of patients with SA. This may be due to the insufficient number of cases and/or the similarity of patient statuses (Fig. 5 and Table I). The present study examined the effect of FN1 on the proliferation and apoptosis of trophoblasts by CCK-8 and flow cytometric assays. The results revealed that the silencing of FN1 significantly decreased cell viability and promoted cell apoptosis. By contrast, FN1 overexpression significantly increased cell viability and inhibited apoptosis (Fig. 5). Caspase-3, Bax and Bcl-2 are critical apoptosis-related genes (32), and studies have demonstrated that Bcl-2 and Bax not only act as upstream regulatory factors of caspase- 3 , but also function as direct substrates of caspase-3. The present experimental results revealed that FN1 silencing significantly upregulated the protein levels of cleaved caspase-3, Bax and Bcl-2, which was consistent with the observed effect of FN1 silencing on cell apoptosis. PCNA and Ki67 reflects the proliferative activity of cells. FN1 silencing significantly reduced the protein levels of PCNA and Ki67, which was consistent with the observed inhibition of cell viability induced by FN1 silencing and the opposite effect of FN1 overexpression (Fig. 6). These results suggest that FN1 regulates the proliferation and apoptosis of trophoblasts.

The PI3K/Akt pathway is an important pathway in the regulation of cell proliferation and apoptosis (33). Akt is a serine/threonine kinase and an important target of PI3K. Activated Akt activates or inhibits its downstream target proteins, such as Bcl-2 and caspase-3, to regulate cell proliferation and apoptosis (34). The results of the present study confirmed that in FN1-silenced cells, the apoptotic levels increased, and the PI3K and Akt phosphorylation levels significantly decreased. By contrast, in FN1-overexpressing cells, the apoptotic levels decreased, and PI3K and Akt phosphorylation levels significantly increased. This suggests that an activated PI3K/Akt signaling pathway exerts a protective effect on trophoblasts. Further analyses revealed that the effects of FN1 silencing on cell apoptosis were reversed by a PI3K/Akt signaling pathway agonist, while the effect of FN1 overexpression on cell apoptosis was reversed by a PI3K/Akt signal pathway inhibitor (Fig. 7). These results suggest that FN1 inhibits trophoblast apoptosis by activating the PI3K/Akt signaling pathway. The results of the animal experiment revealed that FN1 knockdown damaged the organization of the chorionic villus tissues in NP mice and induced cleaved caspase-3 (Fig. 8). More importantly, FN1 knockdown increased the SA-induced apoptosis of trophoblasts and exacerbated chorionic villus tissue injury. Thus, FN1 downregulation was detected in patients with SA and promoted the SA-induced apoptosis of trophoblasts. In addition, FN1 knockdown induced trophoblast cell apoptosis in NP mice. These results revealed that FN1 plays an important role in maintaining the dynamic balance of proliferation and apoptosis of trophoblasts during pregnancy. However, it is worth noting that FN1 has been reported to be upregulated in cervical cancer tissues and to promote cell proliferation (35). Therefore, the results of the present study only suggest that the overexpression of FN1 may be helpful for inhibiting the SA-induced apoptosis of trophoblasts, but do not indicate that the overexpression of FN1 would be helpful in other diseases.

In conclusion, the present study demonstrated that FN1 expression was significantly downregulated in the chorionic villus tissues of patients diagnosed with and mice subjected to SA. The silencing of FN1 promoted trophoblast apoptosis and inhibited cell viability; the overexpression of FN1 inhibited trophoblast apoptosis and promoted cell viability. The activation of the PI3K/Akt signaling pathway protected the trophoblasts. The overexpression of FN1 inhibited apoptosis and promoted cell viability by activating the PI3K/Akt signaling pathway.

\section{Acknowledgements}

Not applicable.

\section{Funding}

The present study was supported by the scientific research project youth project of Nantong's Health and family planning commission (no. QA2019005).

\section{Availability of data and materials}

The GSE127170 dataset was used to perform the present study (https://www.ncbi.nlm.nih.gov/geo/query/acc. cgi?acc $=$ GSE127170). All data generated and/or analyzed during the present study are included in this article.

\section{Authors' contributions}

$\mathrm{JJ}$ and LC wrote the main manuscript and analyzed the data. JJ, LC, YZ and YH performed all the experiments and collected 
the data. WT and FX conceived and designed the study. All authors read and approved the final manuscript and agree to be accountable for all aspects of the research in ensuring that the accuracy or integrity of any part of the work are appropriately investigated and resolved.

\section{Ethics approval and consent to participate}

The study protocol was approved by the ethics committee of the First Affiliated Hospital of Soochow University. A written informed consent was obtained for each sample, which was then analyzed anonymously. This study was performed in accordance with the Declaration of Helsinki. All of the experiments involving animals in this study were carried out in accordance with the protocols of the Guidelines for the Care and Use of Laboratory Animals published by the United States National Institutes of Health (NIH Publication, revised 2011) and the Guidelines for the Care and Use of Laboratory Animals of the Chinese Animal Welfare Committee. The procedures were approved by the Animal Use Committees of Soochow University.

\section{Patient consent for publication}

Not applicable.

\section{Competing interests}

The authors declare that they have no competing interests.

\section{References}

1. Moser G, Windsperger K, Pollheimer J, de Sousa Lopes SC and Huppertz B: Human trophoblast invasion: New and unexpected routes and functions. Histochem Cell Biol 150: 361-370, 2018.

2. Takahashi H, Ogoyama M, Nagayama S, Suzuki H, Ohkuchi A, Matsubara S and Takizawa T: Extravillous trophoblast invasion accelerated by WNT3A, 5A, and 10B via CD44. J Matern Fetal Neonatal Med: 1-9, 2019.

3. Walentin K, Hinze C and Schmidt-Ott KM: The basal chorionic trophoblast cell layer: An emerging coordinator of placenta development. Bioessays 38: 254-265, 2016.

4. Ohgaki R, Ohmori T, Hara S, Nakagomi S, Kanai-Azuma M, Kaneda-Nakashima K, Okuda S, Nagamori S and Kanai Y: Essential roles of L-Type amino acid transporter 1 in syncytiotrophoblast development by presenting fusogenic $4 \mathrm{~F} 2 \mathrm{hc}$. Mol Cell Biol 37: e00427-16, 2017.

5. Gu Y, Zhao S, Wan J, Meng J, Zuo C, Wang S, Zhou Y, Li H and Wang X: Hsa-miRNA-125b may induce apoptosis of HTR8/SVneo cells by targeting MCL1. Reprod Biol 19: 368-373, 2019.

6. Ding J, Yin T, Yan N, Cheng Y and Yang J: FasL on decidual macrophages mediates trophoblast apoptosis: A potential cause of recurrent miscarriage. Int J Mol Med 43: 2376-2386, 2019.

7. Liu HN, Tang XM, Wang XQ, Gao J, Li N, Wang YY and Xia HF MiR-93 inhibits trophoblast cell proliferation and promotes cell apoptosis by targeting BCL2L2 in recurrent spontaneous abortion. Reprod Sci 27: 152-162, 2020.

8. Tian S, Yu J, Zhang Y, Bian Y, Ma J and Yan J: Overexpression of PTEN regulated by miR-19b and miR-494 in the villous of recurrent spontaneous abortion patients. J Reprod Immunol 140: 103133, 2020.

9. Yang D, Ding J, Wang Y, Yuan M, Xian S, Zhang L, Liu S, Dai D, Wang F, Zheng Y, et al: YY1-PVT1 affects trophoblast invasion and adhesion by regulating mTOR pathway-mediated autophagy. J Cell Physiol 235: 6637-6646, 2020.

10. Xu TP, Huang MD, Xia R, Liu XX, Sun M, Yin L, Chen WM, Han L, Zhang EB, Kong R, et al: Decreased expression of the long non-coding RNA FENDRR is associated with poor prognosis in gastric cancer and FENDRR regulates gastric cancer cell metastasis by affecting fibronectin1 expression. J Hematol Oncol 7: 63, 2014.
11. Chen Z, Tao Q, Qiao B and Zhang L: Silencing of LINC01116 suppresses the development of oral squamous cell carcinoma by up-regulating microRNA-136 to inhibit FN1. Cancer Manag Res 11: 6043-6059, 2019.

12. Dong Z, Lin C, Liu Y, Jin H, Wu H, Li Z, Sun L, Zhang L, Hu X, Wei Y, et al: Upregulation of sestrins protect atriums against oxidative damage and fibrosis in human and experimental atrial fibrillation. Sci Rep 7: 46307, 2017.

13. Satoh A, Niwano S, Niwano H, Kishihara J, Aoyama Y, Oikawa J, Fukaya $\mathrm{H}$, Tamaki $\mathrm{H}$ and Ako J: Aliskiren suppresses atrial electrical and structural remodeling in a canine model of atrial fibrillation. Heart Vessels 32: 90-100, 2017.

14. Tian L, Lu ZP, Cai BB, Zhao LT, Qian D, Xu QC, Wu PF, Zhu Y, Zhang JJ, Du Q, et al: Activation of pancreatic stellate cells involves an EMT-like process. Int J Oncol 48: 783-792, 2016.

15. Wang Y, Fu Y, Yan Z, Zhang XB and Pei M: Impact of fibronectin knockout on proliferation and differentiation of human infrapatellar fat pad-derived stem cells. Front Bioeng Biotechnol 7: 321, 2019.

16. Hou J, Li L, Zhu H, Chen H, Wei N, Dai M, Ni Q and Guo X: Association between breast cancer cell migration and radiosensitivity in vitro. Oncol Lett 18: 6877-6884, 2019.

17. Gao R, Feng Q and Tan G: microRNA-613 exerts anti-angiogenic effect on nasopharyngeal carcinoma cells through inactivating the AKT signaling pathway by down-regulating FN1. Biosci Rep 39: BSR20182196, 2019.

18. Zhang S, Wang Q, Han Q, Han H and Lu P: Identification and analysis of genes associated with papillary thyroid carcinoma by bioinformatics methods. Biosci Rep 39: BSR20190083, 2019.

19. Litovkin KV, Ivanova OV, Bauer A, Hoheisel JD, Bubnov VV and Zaporozhan VN: Microarray study of gene expression in uterine leiomyoma. Exp Oncol 30: 106-111, 2008.

20. Mu Y, Zhou DN, Yan NN, Ding JL and Yang J: Upregulation of ADAMTS7 and downregulation of COMP are associated with spontaneous abortion. Mol Med Rep 19: 2620-2626, 2019.

21. Lu X, Cui J, Cui L, Luo Q, Cao Q, Yuan W and Zhang H: The effects of human umbilical cord-derived mesenchymal stem cell transplantation on endometrial receptivity are associated with Th1/Th2 balance change and uNK cell expression of uterine in autoimmune premature ovarian failure mice. Stem Cell Res Ther 10: 214, 2019.

22. Livak KJ and Schmittgen TD: Analysis of relative gene expression data using real-time quantitative PCR and the 2(-Delta Delta C(T)) method. Methods 25: 402-408, 2001.

23. Zipori Y, Haas J, Berger H and Barzilay E: Multifetal pregnancy reduction of triplets to twins compared with non-reduced triplets: A meta-analysis. Reprod Biomed Online 35: 296-304, 2017.

24. Lorek D, Kedzierska AE, Slawek A and Chelmonska-Soyta A: Expression of Toll-like receptors and costimulatory molecules in splenic B cells in a normal and abortion-prone murine pregnancy model. Am J Reprod Immunol 82: e13148, 2019.

25. Xu WM, Xiao ZN, Wang XB and Huang Y: IL-17 induces fetal loss in a CBA/JxBALB/c mouse model, and an anti-IL-17 antibody prevents fetal loss in a CBA/JxDBA/2 mouse model. Am J Reprod Immunol 75: 51-58, 2016.

26. Ciaramella V, Sasso FC, Di Liello R, Corte CM, Barra G, Viscardi G, Esposito G, Sparano F, Troiani T, Martinelli E, et al: Activity and molecular targets of pioglitazone via blockade of proliferation, invasiveness and bioenergetics in human NSCLC. J Exp Clin Cancer Res 38: 178, 2019.

27. Moon JR, Oh SJ, Lee CK, Chi SG and Kim HJ: TGF- $\beta 1$ protects colon tumor cells from apoptosis through XAF1 suppression. Int J Oncol 54: 2117-2126, 2019.

28. TangRZ,GuSS,Chen XT,HeLJ,Wang KPandLiuXQ:Immobilized transforming growth factor-Beta 1 in a stiffness-tunable artificial extracellular matrix enhances mechanotransduction in the epithelial mesenchymal transition of hepatocellular carcinoma. ACS Appl Mater Interfaces 11: 14660-14671, 2019.

29. Cox J, Malik M, Britten J, Lewis T and Catherino WH: Ulipristal acetate and extracellular matrix production in human leiomyomas in vivo: A laboratory analysis of a randomized placebo controlled trial. Reprod Sci 25: 198-206, 2018.

30. Graubner FR, Boos A, Aslan S, Kucukaslan I and Kowalewski MP: Uterine and placental distribution of selected extracellular matrix (ECM) components in the dog. Reproduction 155: 403-421, 2018.

31. Szostek-Mioduchowska AZ, Lukasik K, Skarzynski DJ and Okuda $\mathrm{K}$ : Effect of transforming growth factor $-\beta 1$ on alpha-smooth muscle actin and collagen expression in equine endometrial fibroblasts. Theriogenology 124: 9-17, 2019. 
32. Hao G, Zhai J, Jiang H, Zhang Y, Wu M, Qiu Y, Fan C, Yu L, Bai S, Sun L and Yang Z: Acetylshikonin induces apoptosis of human leukemia cell line K562 by inducing S phase cell cycle arrest, modulating ROS accumulation, depleting Bcr-Abl and blocking NF- $\mathrm{BB}$ signaling. Biomed Pharmacother 122: 109677, 2019.

33. Zhang C, Liang R, Gan X, Yang X, Chen L and Jian J: MicroRNA-384-5p/Beclin-1 as potential indicators for epigallocatechin gallate against cardiomyocytes ischemia reperfusion injury by inhibiting autophagy via PI3K/Akt pathway. Drug Des Devel Ther 13: 3607-3623, 2019.
34. Lv CL,Zhang T, Yan TZ,YiGK and Gao K: MicroRNA-448 inhibits the regeneration of spinal cord injury through PI3K/AKT/Bcl-2 axis. Eur Rev Med Pharmacol Sci 23: 2719-2726, 2019.

35. Wang S, Gao B, Yang H, Liu X, Wu X and Wang W: MicroRNA-432 is downregulated in cervical cancer and directly targets FN1 to inhibit cell proliferation and invasion. Oncol Lett 18: 1475-1482, 2019.

(c) (1) () This work is licensed under a Creative Common Attribution-NonCommercial-NoDerivatives 4.0 International (CC BY-NC-ND 4.0) License. 